Revue d'histoire de l'Amérique française

ATS REVUE D.HISTOIRE DE L'AMÉRIQUE FRANÇAISE

\title{
Essai sur les finances canadiennes (1700-1750) (suite et fin)
}

\section{Guy Frégault}

Volume 13, numéro 2, septembre 1959

URI : https://id.erudit.org/iderudit/301966ar

DOI : https://doi.org/10.7202/301966ar

Aller au sommaire du numéro

Éditeur(s)

Institut d'histoire de l'Amérique française

ISSN

0035-2357 (imprimé)

1492-1383 (numérique)

Découvrir la revue

Citer cet article

Frégault, G. (1959). Essai sur les finances canadiennes (1700-1750) (suite et fin). Revue d'histoire de l'Amérique française, 13(2), 157-182.

https://doi.org/10.7202/301966ar d'utilisation que vous pouvez consulter en ligne.

https://apropos.erudit.org/fr/usagers/politique-dutilisation/ 


\section{ESSAI SUR LES FINANCES CANADIENNES * (1700-1750)

\author{
(suite et fin)
}

\section{FINANCES DE GUERRE: 1744-1751}

En 1744, le Canada entre dans une période d'économie de guerre qui ne prendra fin qu'avec le régime, au moment de la Conquête. Le conflit et la paix armée qui va suivre avant la reprise des dernières hostilités coûteront extrêmement cher. Les dépenses auxquelles la métropole consent avec peine, quand elle y consent, pour donner du muscle à la collectivité américaine qu'elle a lancée dans l'histoire, il lui faut bien se résoudre à les accepter lorsque l'ennemi menace de lui faire perdre la possession même de la colonie. Les grosses sommes qu'elle va mettre dans les opérations militaires se répandront en grande partie dans le commerce canadien. Celui-ci en recevra une forte impulsion. L'argent roulera en abondance dans la société. «La guerre, observe Bougainville en 1757, enrichit le Canada; avant la guerre de 1741 [1744], le Canada devait trois ou quatre cent mille francs à la France, et à la fin de la guerre la France lui devait plus d'un million.» ${ }^{62}$ Négligeons les chiffres que donne le grand voyageur; mais retenons son idée, elle est juste: les dépenses de guerre vont introduire dans la machine un carburant qui la fera tourner plus vite que jamais.

L'accroissement rapide et continu des dépenses semble avoir pris tout le monde au dépourvu: depuis l'intendant, qui s'en excuse, jusqu'au ministre de la Marine, qui s'en irrite. Seul, La Galissonière paraît avoir trouvé le mot juste lorsqu'il explique, non sans brusquerie: «La guerre ne se fait nulle part sans

* Voir notre Revue, XII : 307-322, 459-484 ; XIII : 30-44. 64.

62 «Mémoire sur l'état de la Nouvelle-France», RAPQ (1922-1923), 
dépense.» ${ }^{63}$ Ce qui lui attire cette réplique où perce l'impatience de la Cour: "On n'ignore point que la guerre ne se fait point sans qu'il en coûte; et c'est principalement pour cette raison que le Roy ne peut pas la faire partout. $\mathrm{Sa} \mathrm{Ma}^{\text {té }}$ desire donc que vous vous en teniés aux dispo ${ }^{\text {ons }}$ et aux mouvemens que vous jugerez necessaires pour mettre le Canada a couvert des entreprises que les Ennemis pourroient y tenter...» ${ }^{64}$ En d'autres termes, le Canada doit se borner à une défensive peu coûteuse, éviter toute offensive dispendieuse.

Mais n'est-ce point précisément la ligne de conduite qu'adopte la colonie au cours de ces années de conflit ? Du côté de l'Acadie, où il y eut des expéditions importantes - d'abord celle de Marin en 1745, puis celle de Ramezay, qui devait opérer en liaison avec le duc d'Anville, - c'était toujours la défense de la principale colonie du Saint-Laurent qu'il s'agissait, aux yeux des chefs, d'organiser. Il importe, écrivent Beauharnais et Hocquart en 1745 , de « reprendre Louisbourg et faire la Conquete de l'Acadie, La Conservation du Canada en depend $\gg .{ }^{65}$ L'entretien du détachement de Marin coûta 93.000 livres; ${ }^{66}$ la même année 1745, le ravitaillement de l'île Royale occasionna au gouvernement canadien une dépense de 86.000 livres. ${ }^{67}$ L'année suivante, l'envoi d'un corps de 1.500 hommes en Acadie contraindra le pays à des débours de plus de 200.000 livres: ${ }^{68}$ à lui seul, le transport du détachement reviendra à 65.000 livres, et les appointements des fonctionnaires civils attachés à sa suite se chiffreront par 8.000 livres. ${ }^{69}$ En 1747, les opérations militaires qui se poursuivent en Acadie entraînent une nouvelle mise de fonds de près de 200.000 livres. ${ }^{70}$

63 François Bigot, administrateur français, 2: 167.

64 Ibid., 1: 133.

65 Beauharnais et Hocquart à Maurepas, 12 septembre-7 octobre 1745, AC, C 11A, 83: 3v-4.

$6 \ddot{B}$ Hocquart à Maurepas, 2 novembre 1745, AC, C 11A, 84: 103.

67 Hocquart à Maurepas, 6 novembre 1745, AC, C 11A, 84: 187v.

68 « Bordereau général de La Recette et Dépense faite en Canada pendant L'année 1746 », 24 octobre 1751, AC, C 11A, 115: 316v-317, 326v-327. 69 Hocquart à Maurepas, 6 novembre 1746, AC, C 11A, 86: 52v. Voir «Depenses concernant L'Acadie. Exercice 1746 », AC, C 11A, 85: 373v-374. 70 « Dépense a L'occasion de la guerre 1747 》, AC, C 11A, 88: 213. 
A ces «mouvements 》 et à ceux qui se déroulent en même temps dans les pays d'en haut, il faut employer des indigènes: c'est-à-dire acheter leur fidélité, acheter leurs services, acheter - ce sera le cas des Iroquois - jusqu'à leur neutralité. Prenons le cas des Indiens «domiciliés», ainsi qu'on désigne ceux que le gouvernement et les missionnaires ont groupés en villages à l'intérieur de la colonie. Ils ont toujours maintenu des liaisons de commerce avec les Anglais. Il faut les empêcher de passer à l'ennemi. On s'y appliqua dès 1744, raconte Hocquart, «mais ce fût avec une quantité de présens si considerable que L'article seul de la Porcelaine pour colliers acheptée cette même année... monta a 10.091 livres 16 sols, indépendament de ce qui pouvoit Estre alors dans les magasins »; et, comme le rappelle l'intendant, la présentation des colliers de porcelaine ne servait qu'à engager les conversations; il fallait ensuite parler à coups de cadeaux de beaucoup plus de valeur. ${ }^{71}$

On lance sur les frontières de la Nouvelle-Angleterre et du New-York des Indiens de l'Acadie, des sauvages domiciliés, d'autres encore que l'on recrute dans l'Ouest. Comme ils abandonnent, pour faire ces courses, leur chasse et leurs cultures, on doit faire vivre non seulement les guerriers, mais aussi leurs familles. C'est ce qui fait que les sauvages coûtent si cher. Mais «il faut avouer, souligne Hocquart en 1746, que jusques a present ils ont infiniment contribué a la sureté de la Colonie dont ils gardent les avenuës; dans la situation ou nous sommes, nous ne pouvons nous sauver, je l'ose dire, qu'a force d'argent $\gg{ }^{72}$ En 1747, des familles indigènes sont campées aux environs de Québec, où elles forment une agglomération de 2.000 âmes: aucun de ces sauvages, qu'il faut nourrir, vêtir, armer, ne coûte au roi, l'un portant l'autre, moins de 250 livres. $^{73}$ « Si, répète l'intendant, nous nous Estions tenus dans L'inaction, et que nous n'Eussions point remué nos Sauvages, Ces derniers En Continuant Leurs liaisons avec Les anglois se seroient a la

71 Hocquart à Maurepas, 24 septembre 1747, AC, C 11A, 88: 16-16v.

72 Hocquart à Maurepas, ler octobre 1746, AC, C 11A, 85: 323v-325.

73 Hocquart à Maurepas, 24 septembre 1747, AC, C 11A, 88: 18v. Voir Hocquart à Maurepas, 9 juillet 1747, $i$ bid., $4 \mathrm{v}$. 
fin Séparez de Nous, ils veulent estre Employez, Les seules caresses n'auroient pas sufi pour nous les Conserver, Les Libéralités et les récompenses Nous les ont attaché et nous En avons tiré de grands services. ${ }^{74}$

Que ce ne soient pas les démonstrations d'amitié - les «caresses»- qui gagnent aux Canadiens le concours des indigènes, on le voit à plus d'une reprise en examinant les états financiers. Voici, à titre d'exemple, ce qui se passe au poste des Ouiatanons de septembre 1746 à mai 1747. Il ne s'agit pas d'un établissement aussi important que le Détroit ou Michilimakinac. Situé sur le cours supérieur de la Wabash, dans l'Indiana actuel, ce poste est fréquenté par des Ouiatanons, des Kikapous, des Mascoutins, des Péanguichias. Le commandant leur verse assez régulièrement des subsides. Voici un chef de bande, il s'appelle «la Teste Blanche»; on lui remet huit bouteilles d'eau-de-vie, dix livres de tabac, trois chemises, deux aunes de drap, une couverture, d'autres articles encore, le tout d'une valeur de 326 livres. Voici un autre chef, celui-ci est surnommé «l'Homme »: on distribue à l'Homme et à sa bande 8 bouteilles d'eau-de-vie, dix livres de tabac, etc., et ces présents valent également 326 livres. Le lendemain, un chef Kikapou se présente, il s'appelle «les Deux visage plats»: lui et sa bande reçoivent à leur tour 326 livres en eau-de-vie, tabac et autres marchandises. Quelques jours plus tard, le commandant donne les mêmes présents à la bande du «Temps clair», chef mascoutin. D'autres chefs viennent toucher leur solde: l'un a nom le Comte, un autre, la Peau blanche, un troisième, l'Enfant, un autre encore, le Brave. Le Brave est sans doute un personnage puisqu'au lieu de huit bouteilles d'eau-de-vie, on lui en donne dix, outre le tabac, le vermillon, les frusques qu'il reçoit comme ses congénères. Après le Brave, voici Agathe, puis la Graine, à qui on ne donne que deux livres de tabac et une bouteille d'eau-de-vie. Il y a encore la Mauvaise jambe, la Petite jarretière, le Commis qui ne sont pas oubliés, ainsi que la Noix, le Petit bonheur... On croirait parcourir un bottin de la pègre internationale, où pareils surnoms

74 Hocquart à Maurepas, 27 octobre 1747, ibid., 156. 
sont d'usage. En 1746-47, ces tueurs à gages de la région des Ouiatanons reçurent ainsi pour près de 6.000 livres de présents. ${ }^{75}$

Il ne suffisait pas de se procurer la fidélité de ces «alliés 》. Il fallait aussi rémunérer exactement leur travail. Un prix fixe était attaché aux prisonniers ennemis et aux chevelures qu'ils rapportaient de leurs incursions. En 1748, le gouvernement canadien payait un scalpe 31 livres et un prisonnier, 124. Il versa ainsi, cette année-là, 2.937 livres aux Abénakis de Bécancourt, de Narantsouak et de Panaouamské. ${ }^{76}$

Il reste pourtant que les plus fortes augmentations de dépenses se produisent à l'article des achats de vivres et de munitions; les services des fortifications et de l'artillerie absorbent aussi des sommes considérables (tableau IV).

TABLEAU IV

\section{DÉPENSES MILITAIRES}

\begin{tabular}{|c|c|c|c|c|}
\hline \multirow{2}{*}{$\begin{array}{l}\text { Année } \\
1744\end{array}$} & \multicolumn{2}{|c|}{$\begin{array}{l}\text { Achats de vivres } \\
\text { et marchandises }\end{array}$} & \multicolumn{2}{|c|}{$\begin{array}{c}\text { Fortifications et } \\
\text { artillerie }\end{array}$} \\
\hline & 380.055 & livres & 111.195 & livres \\
\hline 1745 & 777.736 & 6 & 245.170 & "6 \\
\hline 1746 & 1.527 .423 & “" & 263.266 & "6 \\
\hline 1747 & 1.188 .314 & “" & 302.100 & 6 \\
\hline 1748 & 547.399 & “ & 403.548 & “" \\
\hline 1749 & 687.731 & “ & 307.437 & " \\
\hline 1750 & 1.386 .290 & “ & 216.029 & 66 \\
\hline 1751 & 1.933 .051 & 66 & 242.723 & 6 \\
\hline
\end{tabular}

D'où proviennent les fonds nécessaires à l'acquittement de ces dépenses accrues ? D'une augmentation d'impôt ? C'était là une éventualité que le gouvernement métropolitain envisageait depuis des années. Les administrateurs coloniaux réussirent

75 « Estat des fournitures faites par moy Michel Gamelin au Poste des ouyatanons pour le Compte et Service du Roy Suivant Les ordres de M. le Cher Delaperade Commandant pour le Roy aud. Lieu 》, AC, C 11A, 118: $9-13 \mathrm{v}$.

${ }_{76}$ « Canada. Exercice 1748. Bordereau des Dépenses généralles dont les acquits ont été payées par le Trésorier de Quebec, sur les fonds de l'Exercice de L'année MVIIc Quarante huit, depuis Le premier Janvier Jusques au dernier Septembre de la d. année », AC, C 11A, 116: 134-135. 
longtemps à l'écarter. ${ }^{77}$ Mais quand les dépenses de guerre se furent portées à près de trois millions, la Cour prit la décision assez naturelle d'inviter les Canadiens à y contribuer de leurs deniers; ce n'était, après tout, que justice, vu les profits que le commerce colonial réalisait sur les fournitures qu'il faisait à l'État. Ce qui est surprenant, c'est de constater combien peu le roi exigea de ses sujets du Canada.

Lorsque la guerre éclata, il y avait près de trente ans que le gouvernement français avait ordonné à la ville de Montréal de verser un impôt annuel de 6.000 livres - dont 2.000 payables par les Sulpiciens - afin de défrayer l'érection et l'entretien des fortifications dont il était devenu nécessaire d'entourer l'agglomération. ${ }^{78}$ Les Montréalais montrèrent une répugnance entêtée à payer leur «imposition ». En 1743, deux ans après que l'enceinte eut été «mise dans sa perfection», le roi faisait observer qu'il avait avancé 329.617 livres pour les travaux; il fit remise de la moitié de cette somme à la population montréalaise, mais lui enjoignit de continuer à payer tous les ans 6.000 livres jusqu'au parfait recouvrement du reste de la contribution royale. ${ }^{79}$ L'impôt de Montréal devait donc rester en vigueur jusqu'à la fin du régime.

En 1745, la chute de Louisbourg fit réfléchir les Québecois: si les Anglais s'étaient emparés sans trop de mal de la forteresse de l'île Royale, ne pourraient-ils pas enlever avec une égale facilité la capitale de la Nouvelle-France ? L'évêque, les officiers, les bourgeois se prirent à vouloir tout de suite une enceinte de pierre. On l'érigerait, bien entendu, aux frais du roi. Hocquart s'y opposait, Beauharnais hésitait. À la fin, le gouverneur se laissa gagner par les sollicitations du public, il y eut assemblée de notables, et les travaux commencèrent aussitôt. ${ }^{80}$ La Cour, cependant, refusa de s'incliner devant le fait accompli. Si, manda-t-elle à ses représentants, les Québecois désiraient des

77 Voir La Civilisation de la Nouvelle-France, 77-81.

78 « Arrest au sujet de l'enceinte de la ville de Montréal. Extrait des registres du Conseil d'Etat », 5 mai 1716, AC, B 38: 215-216.

79 Déclaration du roi du 1er mai 1743, Edits et ordonnances, 1: 567s.

80 Hocquart à Maurepas, 24 septembre 1745, AC, C 11A, 83: 184-187; Beauharnais et Hocquart à Maurepas, 19 octobre 1745, ibid., 39-40. 
fortifications, ils pouvaient les construire, mais devraient les payer; s'ils ne voulaient pas délier leur bourse, ils n'auraient qu'à démolir les ouvrages mis en chantier. À la fin de juillet 1746, autre assemblée de notables pour mettre le public au courant de la décision de Maurepas et recueillir les avis. L'opinion se partagea nettement: d'une part, l'évêque, l'état-major, les officiers militaires, le chapitre, le Séminaire, les Jésuites se déclarèrent partisans de l'enceinte; de l'autre, des fonctionnaires civils et le bloc des négociants s'y opposèrent. ${ }^{81}$ Les esprits, au dire de l'évêque, étaient échauffés. ${ }^{82}$ Celui du gouverneur général l'était peut-être plus que tout autre. Beauharnais voulait maintenant à toute force des murailles. La résistance venait des bourgeois; il les foudroyait: «C'est donc Esprit de Vertige de la part des dits Bourgeois que de pretendre se deffendre en ne se mettant point en Etat de le pouvoir faire... Non, Monseigneur, je ne crains point de le dire, c'est un esprit qui a sa source dans une cabale populaire fomentée par tout ce qu'il y a de plus mauvais. Les uns souhaitent l'anglois pour être libres de penser et croire tout ce qu'ils voudront, les autres le craignent par Excez et voulant s'Exempter de combattre contre luy et colorant leur manque de Coeur du pretexte honorable d'une retraite glorieuse dans les bois qui doit en faire des Caesars lorsqu'il ne sera plus temps pour la Conservation de la Colonie à Sa Majesté. »83 Quant à Hocquart, il résumait ainsi le débat: "Tous les militaires a l'Exception de M. de Rigaud ont esté pour continuer l'ouvrage commencé, Et d'un autre costé les Bourgeois qui ont Esté appellez ont esté d'avis de la demolition. Si je ne me trompe, ce seroit assez L'avis général de tous les habitans de cette Colonie. » ${ }^{84}$ Plus nombreux à l'assemblée, les militaires l'avaient emporté aux voix. Les travaux continuèrent. Comment en défrayer le coût ? Une taille, opine l'évêque, ferait mauvais effet. Il serait difficile, observe

81 Procès-verbal des assemblées des 26 et 30 juillet 1746, AC, C 11A, 85: 76-78.

82 Pontbriand à Maurepas, 10 novembre 1746, AC, C 11A, 86: 266-266v.

83 Beauharnais à Maurepas, 10 octobre 1746, AC, C 11A, 85: 208v-214.

84 Hocquart à Maurepas, $1^{\text {er }}$ octobre 1746 , ibid., 326v-327. 
l'intendant, de percevoir «une imposition par teste». Un «droit d'octroy» conviendrait, affirme le gouverneur. Et tous de proposer une augmentation des droits d'entrée sur les vins et les alcools; le prélat verrait même d'un bon œil un droit «sur les marchandises de soye». En somme, personne ne propose que les Québecois portent seuls tout le poids d'un impôt, «ce qui paroist juste, écrivent Beauharnais et Hocquart, puisque le principal but qu'on s'est proposé dans L'Entreprise Est la conservation de toute la Colonie $\gg .{ }^{85}$ En ce cas, ajoute Pontbriand, il paraîtrait équitable «que Montreal fut dechargé de ce qu'elle paye pour ses fortifications $\gg{ }^{86}$ Le prélat semble être le seul à $\mathrm{y}$ penser.

La Cour retient tout de suite les propositions des chefs de la colonie. Dès janvier 1747, elle ordonne pour trois ans une augmentation des droits d'entrée sur le vin, l'eau-de-vie et la guildive. ${ }^{87}$ L'année suivante, un édit établit un droit de $3 \%$ sur «toutes les marchandises » qui entreront dans le pays et un pareil droit sur toutes celles qui en sortiront; cette législation frappe 305 articles d'importation et 59 articles d'exportation. ${ }^{88}$ Un mois plus tard, toutefois, le Conseil d'Etat sursoit à l'application de l'édit «tant que la présente guerre durera»; en attendant que la paix soit proclamée au Canada, le Domaine se bornera à percevoir les droits habituels, avec l'augmentation qui y a été portée en janvier $1747 . .^{89}$ Aux revenus que donnent ces impôts, s'ajoutent le produit des congés et celui de la ferme de divers postes de traite.

Il reste à voir ce que recueille le trésor colonial. En 1744, les droits d'entrée sur les vins et les alcools montent à 56.838 livres; les droits de sortie, à 1.400 ; les droits seigneuriaux, à 1.430 ; la ferme de Tadoussac donne 4.500 livres. En tout, le $85: 75$.

85 Beauharnais et Hocquart à Maurepas, 10 octobre 1746, AC, C 11A,

86 Pontbriand à Maurepas, 10 novembre 1746, $\mathrm{AC}, \mathrm{C} 11 \mathrm{~A}, 86: 266 \mathrm{v}$. 1: 589 .

87 Arrêt du Conseil d'Etat du 23 janvier 1747, Edits et ordonnances,

88 Edit de février 1748, ibid., 591-594; «Edit et Tarif des Droits 》, 25 février 1748, ibid., 594-605.

89 Arrêt du 6 mars 1748, ibid., 608v. 
Domaine perçoit ainsi 64.168 livres. $^{90}$ Le bordereau des recettes et des dépenses n'en tient pas compte parce qu'il se rapporte à l'état du roi, dont l'administration du Domaine reste distincte. Ce même bordereau comporte toutefois les recettes suivantes:

vente de 25 congés

6.250 livres

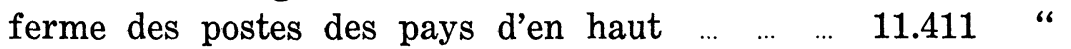

moitié de la ferme des postes de Frontenac et de

Niagara

Total

$\frac{4.000 " “}{21.661 “}$

A cette somme, s'ajoutent les 232.272 livres provenant des ventes effectuées dans les magasins de l'État et les 374.114 livres prévues par l'état du roi, pour former des recettes montant à 628.047 livres. Comme les dépenses atteignent 1.029.404 livres, la Cour devra trouver le moyen de combler le déficit, c'est-à-dire d'apaiser les fournisseurs qui détiennent sur elle des créances se chiffrant par 401.357 livres. Elle y arrive par étapes: le 15 juin 1745, elle destine au paiement de ses dettes une somme de 112.111 livres, suivie, le 29 mars 1746 , d'une autre de 59.181 livres et, le 19 mars 1747, d'une troisième somme de 39.784 livres. Au 30 octobre 1748, le gouvernement royal doit encore 190.281 livres sur les dépenses faites au Canada quatre ans plus tôt. ${ }^{91}$ En 1750 , enfin, il se sera mis en règle à l'égard de ses créanciers. ${ }^{92}$

L'examen des états financiers de 1744 nous enseigne deux choses : d'abord, que le produit des impôts est très faible; ensuite, que la confusion règne dans la comptabilité. La principale cause de cette confusion saute aux yeux: les déficits sont trop importants, et l'État métropolitain met une lenteur excessive à faire

90 « Domaine. Canada. 1744. Extrait du Produit des Droits du Domaine d'occident En Canada Pendant La presente année Mil Sept Cent quarante quatre », AC, C 11A, 82: 204.

91 «Marine 1744. Bordereau des Recettes et Dépenses》, AC, C 11A, 115: 65-66; «Canada. Exercice 1744. Mémoire au sujet de la situation des finances du Canada de L'année 1700 quarante quatre 》, AC, C 11A, $116: 82-86 \mathrm{v}$.

92 «Bordereau des Recettes et Depenses Employées dans le Compte rendu pour l'Exercice 1744 》, AC, C 11A, 115: 119-133v. 
face à ses obligations. Au fait, leur fait-il face? On dirait plutôt qu'il leur présente le dos. Mais elles le rattrapent.

Il n'y aurait guère d'utilité à étudier en détail les états des années suivantes. Il est plus instructif d'examiner le mouvement des impôts à compter du moment où la métropole les augmente. En 1747, la hausse des droits d'entrée sur les boissons produit 24.291 livres. ${ }^{93}$ En 1748, ce même supplément de revenus se porte à 33.907 livres. ${ }^{94}$ L'année suivante, la guerre est finie, le Domaine prélève les droits de $3 \%$ prévus au début de 1748 . Il encaisse ainsi 117.219 livres, somme à laquelle il faut ajouter 45.622 livres levées sur les boissons, 12.931 sur la population québecoise pour la construction de casernes et 6.000 sur Montréal pour les fortifications de cette place; soit, en tout, 181.232 livres: c'est $38.2 \%$ du budget, mais seulement $8.9 \%$ des dépenses..$^{95}$ Pour l'année 1750, il y a lieu de croire que les droits ont pu faire rentrer au trésor quelque 140.760 livres..$^{96}$ En 1751, ils produisent, semble-t-il, 81.145 livres. ${ }^{97}$ Ce chiffre est bas; sans doute le rendement des anciens droits n'y est-il pas compris. Quoi qu'il en soit, il ressort de ce qui précède que les Canadiens n'ont toujours payé que des impôts modiques, même dans les années où la France déclarait son intention de les faire contribuer davantage au coût de l'administration de leur pays.

Puisque les dépenses augmentent sans que les revenus s'accroissent, il est naturel que l'intendant éprouve encore plus de mal qu'avant la guerre à joindre les deux bouts. Nous savons

93 « Marine 1747. Bordereau des Recettes et Dépenses employées dans le compte de l'Exercice de $1747 \gg, A C, C$ 11A, 116: 2.

94 « Extrait du montant des Nouveaux Droits perçûs au Bureau du Domaine d'occident », AC, C 11A, 116: 76.

95 «Bordereau Général de Recette et Dépense faittes en Canada pendant l'année 1749 », AC, C 11A, 116: 214-217.

96 Le bordereau de l'année 1750 , dressé le 12 août 1752 , comporte des recettes extraordinaires s'élevant à 471.871 livres (AC, C 11A, 119: 333). Un autre bordereau de l'année 1750, dressé le 26 octobre 1751, établit les recettes extraordinaires à 331.111 livres et précise que le produit des impôts n'y est pas compris, "Les Commerçants n'ayant pas eu de quoy y Satisfaire Entierement 》 (ibid., 372-374). La différence entre ces deux chiffres provient peut-être de ce que le bordereau compilé en 1752 tient compte des impôts rentrés.

97 « Bordereau général de Recette et dépense faites en Canada pendant L'année mil sept cent cinquante-un », AC, C 11A, 119: 406-408. 
que la métropole mit des années à éteindre les créances canadiennes de 1744. Néanmoins, cette année-là, Hocquart put acquitter toutes les écritures qui rentrèrent à la caisse en tirant pour 521.372 livres de lettres de change, à peine plus que les années précédentes. ${ }^{98}$

Il en alla autrement l'année suivante. D'abord, on ne rapporta au trésor que pour 50.000 livres de monnaie de cartes. Afin de subvenir aux besoins du service, l'intendant avait dû se servir presque exclusivement de billets. Il en reflua à la caisse pour plus d'un million. Hocquart en fit convertir la totalité en lettres de change sur Paris. De cette façon, déclara-t-il à la Cour, le crédit du roi «s'est bien soutenû, mais il est de la derniere Consequence que vous ayez, Monseigneur, pour agreable de faire payer régulierement Les Lettres [de change], sans quoy le service seroit dans le plus grand danger par la perte de toute Confiance ». On comprend que le magistrat ait jugé utile d'entourer son rapport de précautions oratoires: il venait d'autoriser pour 1.084.872 livres de traites, et encore n'en avait-il fait aucune pour les dépenses de l'Acadie ni pour celles des pays d'en haut, et il n'en avait distribué que pour 31.000 livres à compte des dépenses de l'île Royale, qu'il savait monter à 86.000 livres. ${ }^{99}$

En 1746, les dépenses sont «immenses », annonce Hocquart à la mi-septembre. Elles sont «prodigieuses», reprend-il au début d'octobre. Il explique: il a fallu «faire achepter a gros frais » les munitions nécessaires pour tenir en campagne de nombreux détachements formés de Canadiens et de sauvages; l'expédition d'Acadie coûte cher; enfin, le haut prix des marchandises « a Encor doublé la dépense ». Tous les achats se font en billets. L'intendant déclare à ce propos: «La confiance que l'on a icy dans le papier m'a beaucoup aydé. Si le Credit venoit a s'en perdre par quelque empeschement que ce fût, je me trouverois dans l'Impossibilité absoluë de faire face au service.»

98 Hocquart à Maurepas, 2 novembre 1744, AC, C 11A, 82: 243-244. 99 Hocquart à Maurepas, 6 novembre $1745, \mathrm{AC}, \mathrm{C} 11 \mathrm{~A}, 84$ : $187-188 \mathrm{v}$; «Etat des lettres de change Tirées ... sur M. Louis Barthelemy Moufle de Georville ... 》, 30 octobre 1745, ibid., 196-198; "Etat des lettres de change tirées .... sur M. Marcellin François Zacharie de Selle...», 30 octobre 1745, ibid., 200-205. 
Aussi s'est-il cru tenu de tirer des lettres de change pour tout le montant des engagements qu'on lui a présentés, et ceux-ci se sont chiffrés par 2.093.221 livres. Ce n'est pas tout: il s'est effectué aussi d'autres dépenses, mais on n'en connaît pas le total, une partie des factures n'étant pas rentrées. ${ }^{1}$

Cette fois, la Cour ne ménage pas les observations désagréables à son représentant. Il a tiré beaucoup trop de lettres de change, tranche Maurepas; les dépenses qu'elles représentent sont trop élevées pour qu'il ne se soit pas commis «d'abus» quelque part. «Quoiqu'il en soit, enchaîne le ministre, j'ignore si ces traittes pourront estre acquittées ... Mais en tout cas, je dois vous prevenir que la Caisse de la Marine seroit dans l'impossibilité absolüe de supporter de pareilles charges pour l'année prochaine. $\gg^{2}$ Les remarques désobligeantes du ministre et plus encore l'incertitude qu'il laisse planer sur le paiement des lettres de change ne manquent pas d'impressionner Hocquart. Il y fait allusion à deux reprises pour s'expliquer, presque pour se défendre, mais il peut seulement prévoir que les dépenses de 1747 «seront au moins aussi Considerables » que celles de l'année précédente. ${ }^{3}$

Effectivement, en 1747 , le magistrat fait tirer pour 2.063 .178 livres de lettres de change. Ce n'est pas de gaieté de cœur. Pour éviter de dépasser cette somme, il a dû, sans aucun doute, renvoyer insatisfaits beaucoup de créanciers puisque, du 25 septembre au 10 octobre, on a rapporté à sa caisse $\mathbf{5 5 . 3 9 2}$ livres en monnaie de cartes et 2.669.358 livres en billets du trésor. Quelles dépenses! «Ce n'est pas ma faute, proteste-t-il, si Elles sont prodigieuses.» Il faut en chercher la cause dans l'ampleur des

1 Hocquart à Maurepas, 18 septembre 1746, AC, C 11A, 85: 310; id. à $i d ., 1^{\text {er }}$ octobre 1746 , ibid., $323 \mathrm{v}-324$; $i d$. à $i d$., 6 novembre 1746 , AC, C $11 \mathrm{~A}$, 86: 51-54; «Estat des Lettres de change tirées... sur M. de Georville 》, 5 novembre 1746, AC, C 11A, 85: 369-374; «Estat des lettres tirées ... sur M. De Selle 》, 5 novembre 1746, AC, C 11A, 86: 38-48; «Construction des vaisseaux du Roy 》, 5 novembre 1746, ibid., 49v-50; «Etat de sept lettres tirées triples sur Monsieur de Selle... en addition acompte 1747 », 12 novembre 1746 , ibid., 3 .

2 Maurepas à Hocquart, 23 janvier 1747, Shortt, 2: 746-748.

3 Hocquart à Maurepas, 9 juillet 1747, AC, C 11A, 88: $3-4 \mathrm{v}$; id. à id., 24 septembre 1747 , ibid., 18-22. 
opérations militaires. Aussi l'intendant insiste-t-il sur le tour heureux que celles-ci ont pris: "Nos succez parlent, il y en a Eû même quelques uns de brillans... $\gg^{4}$ Il reste qu'il a fallu les acheter à prix d'or, ou plutôt de ce qui en tient lieu. Le gouvernement colonial n'aurait-il pas pu payer en monnaie de cartes une bonne partie des frais qu'entraîne le conflit? Hocquart a prévu l'objection. Les 720.000 livres de cartes qui circulent dans le pays, rappelle-t-il, «n'ont pû servir a l'acquittement de ces dépenses, il y a desja plusieurs années qu'Elles ont Esté Employées pour celles des anciens Exercices ». Soucieux d'en maintenir le crédit, l'intendant s'empresse de convertir en traites le peu qui en rentre au trésor, vers la fin de chaque année. Il ne peut se dispenser de racheter de même les billets qui y refluent en même temps que les cartes: «D'En user autrement, tout le credit auroit cessé dans le moment et il ne me seroit resté aucun moyen de faire le service.» De peur de submerger la caisse de la Marine sous un flot de lettres de change canadiennes, Hocquart a pris le parti d'en fractionner les échéances et d'espacer celles-ci le plus possible. Cette précaution a créé dans la colonie une impression pénible. Plusieurs se sont imaginé que les traites sur la Compagnie des Indes étaient plus sûres que celles que l'intendant tirait sur la Marine et ils «ont fait des négociations usuraires pour en avoir par préférence ». D'autres ont réalisé tout de suite leurs lettres de change en pelleteries, quittes à perdre $15 \%$ ou même $20 \%$ dans l'opération. ${ }^{5}$

Les inquiétudes des négociants ne sont que trop justifiées. Ces échéances éloignées, exposent-ils dans un mémoire collectif, ruinent le commerce canadien. Les fonds sur lesquels roulent maintenant leurs transactions «sont dehors depuis 1745 ». En conséquence des nouvelles échéances, ils peuvent disposer tout au plus d'un quart de leurs capitaux. Que n'escomptent-ils, se demandera-t-on, les instruments de crédit que leur a fait délivrer

${ }^{4}$ Hocquart à Maurepas, 31 octobre 1747, AC, C 11A, 88: 184-198; «Dépense a L'occasion de la guerre 1747. Sur L'Exercice 1748 », 22 octobre 1747, ibid., 213-213v; "Extrait du fonds nécessaire pour Le payement des Marchandises et Munitions fournies dans les Magasins du Roy à Quebec par le $S^{r}$ Claverie, Negociant », 15 janvier 1747, ibid., 7.

5 Hocquart à Maurepas, 27 octobre 1747, AC, C 11A, 88: 155-159. 
l'intendant? S'ils s'y voient contraints, répliquent-ils, «le change ne pourra estre qu'exorbitant ». Déjà, en 1746, on exigeait d'eux $10 \%$ sur les places de Bordeaux et de la Rochelle: quelle perte n'ont-ils pas lieu d'appréhender par suite «des Longs Termes des Lettres de Change de cette année»? Et ils profitent de l'occasion pour se plaindre des «Reductions sur les acquits »dont ils ont souffert au cours des années précédentes. ${ }^{6}$ Outre les inquiétudes qu'ils expriment dans leur mémoire, ajoutent Beauharnais et Hocquart, les négociants «dissimulent La Crainte qu'ils ont que ces Lettres [de change] ne soient pas aussi régulierement acquittées que l'Interest de Leur Commerce et celuy de la Colonie l'Exigent $\gg^{\top}$ Il est essentiel, avait déjà déclaré l'intendant, de maintenir « la confiance publique ». Il n'y a guère de monnaie métallique au Canada. Le commerce n'y roule que sur les cartes et les billets du trésorier. «Si une fois tout ce papier tombe dans le discredit, L'Usure et L'agiot succederont avec Les plus fatales conséquences.» ${ }^{8}$ Une crise du crédit se laissait pressentir. La cause en était toujours la même: l'écart entre les sommes que l'État métropolitain était disposé à engager dans le développement du Canada et celles dont les circonstances réclamaient l'investissement. Dans les derniers mois de 1747, Hocquart se montre bien déterminé à rétrécir cet écart. Son collègue et lui-même ont pris la résolution d'économiser. Il promet à la Cour: "Vous pouvés compter sur un grand changement l'année prochaine. ${ }^{9}$

Le principal changement qui marque l'année 1748 est le remplacement de Hocquart par François Bigot. Ce dernier, on le sait, arrive dans la colonie avec l'intention de profiter de son

6 « A Monseigneur le Comte de Maurepas Ministre et Sécrétaire d'Estat », AC, C 11A, 87: 290-291. Ce mémoire porte les signatures suivantes: Parant, Dumont, Lamorille, Riverin, La Croix, Turpin, Berthelot, Guillimin, Mounier, Revolt, Gautier, Besançon, Dunière, de Voisy, Duperé, Larcher, Voyer, Amiot, Marchand, Liquart, Chabosseau, Poney, Jarnac, Jayat, Dufour, Panet, Voyer, Thouron, Charest, Saint-Ange Charly, J. Perthuis, Dhenin, Leclère, Berlinguet, Mezières, D. Goguet, Portes, J. Berziau, Lamaletie, Havy et Lefebvre; le syndic, Perthuis; le secrétaire, Tasché.

7 Lettre du 6 novembre 1747, AC, C 11A, 87: 286-286r.

8 Hocquart à Maurepas, 24 septembre $1747, \mathrm{AC}, \mathrm{C} 11 \mathrm{~A}, 88: 26$.

9 Id. à $i d$., 3 novembre $1747, \mathrm{AC}, \mathrm{C} 11 \mathrm{~A}, 89: 23 \mathrm{v}$. 
poste pour faire un commerce avantageux. Au moment où son navire le dépose au Canada, il a en poche un contrat de société avec la grande maison bordelaise d'Abraham Gradis. Ce n'est pourtant pas ce contrat qui introduit Gradis dans le commerce canadien: l'armateur a expédié une cargaison à Québec dès $1747 .{ }^{10}$ Sans être un administrateur modèle, le nouvel intendant a horreur de la «confusion». Ce trait de caractère lui fait prendre tout de suite deux mesures opportunes.

En premier lieu, il constate qu'il existe une marge fantastique entre l'état du roi et les dépenses réelles du pays. Comme il avait fait à l'île Royale à l'époque où il y était commissaireordonnateur, il décide de préparer lui-même le projet d'état du roi, et non pas d'abandonner cette formalité à un subordonné. Dressé avec soin, en tenant compte des dépenses des années précédentes, cet état de prévision sera enfin réaliste. ${ }^{11}$ Voire ! Le budget de 1749 sortira des bureaux de Versailles avec une augmentation d'à peine 20.000 livres sur celui de 1748 , notoirement insuffisant. Celui de 1750 sera plus faible de 40.000 livres que celui de l'année précédente.

Si le nouvel intendant ne parvient pas à faire adopter des états de prévision qui prévoient réellement les dépenses, il réussit, en revanche, à mettre de l'ordre dans la comptabilité du gouvernement canadien. À son arrivée, il y a longtemps que les comptes traînent. En 1745, Hocquart s'était excusé de n'être pas prêt à expédier le compte de 1742, alléguant «les affaires extraordinaires $\gg . .^{12}$ Trois ans après, quand il s'en va, le bilan de cette même année 1742 vient tout juste d'être terminé. Bigot mettra deux mois à rattraper tout ce temps perdu. Dès le début de novembre 1748, il a sous les yeux un tableau assez précis des six exercices consécutifs à 1742. Ce tableau révèle un déficit considérable, formé par une masse croissante d'acquits rejetés d'une année sur l'autre. Il se présente comme suit:

10 « Fonds 1747 Quebec. Etat des fonds a remettre au Port de Rochefort pour le payement du fret des Munitions et marchandises embarquées sur... la Renommée de Bordeaux apartenant au $\mathrm{S}^{\mathrm{r}}$ Gradix .... , AC, C $11 \mathrm{~A}, 89: 346-349$.

11 François Bigot, administrateur français, 1: 329s.

12 Hocquart à Maurepas, 2 novembre 1745, AC, C 11A, 84: 107-107v. 
1743

\begin{tabular}{|c|c|c|}
\hline & ves & $8 \mathrm{s.} 90$ \\
\hline 190.2 & “" & $10 "$ \\
\hline & “ & $1 " 2$ \\
\hline 502. & “ & $11^{\prime \prime} 11^{\prime}$ \\
\hline 966 & “ & $14 " 8$ \\
\hline 128.4 & “ & 4 “ 3 \\
\hline & & \\
\hline
\end{tabular}

Ce total représente les excédents de dépenses non mentionnés dans les bordereaux expédiés à la Cour depuis $1743 .{ }^{13}$ Ceux-ci étaient donc devenus de plus en plus fautifs avec les années. Dressé un peu trop rapidement, le tableau précédent n'est pas tout à fait exact. Deux ans plus tard, l'intendant confectionne plus à loisir des états relatifs aux exercices de 1746 à 1749. C'est encore une liste de déficits:

1746

1747

1743

1749
501.246 livres $12 \mathrm{~s}$.

\begin{tabular}{rcc}
1.003 .116 & “ & 18 “ \\
105.853 & “ & 15 \\
1.324 .244 & “ & 14 \\
\hline 2.934 .461 & “ & 19 “
\end{tabular}

La différence entre les deux tableaux pour ce qui concerne les chiffres de 1748 s'explique par les «suppléments de fonds» que la Cour a ordonnés en 1749 et en 1750 pour réduire la dette contractée par le roi. Depuis son arrivée, Bigot avait adopté un système: expédier les ordonnances en forme au fur et à mesure que les dépenses se font, ce qui, promet-il, «sera fidellement observé par la Suitte $\gg .^{14}$ Ainsi, les comptes cesseront d'être en retard.

Mais les dépenses ne cesseront pas d'être considérables. Celles de 1748 et de 1749 le seront, certes, sensiblement moins

13 François Bigot, administrateur français, 1: $328 \mathrm{~s}$.

14 « Etat des fonds faits par les Etats du Roy du montant des Recettes extraordinaires et des depenses faites en Canada sur les Exercices de 1746. 47. 48. et 49 pour servir a Constater les Excedents de Depenses de chacun de ces Exercices », 24 octobre 1750, AC, C 11A, 116: 238-245. 
que celles de 1747. Elles ne diminueront toutefois pas au point que l'intendant puisse réaliser la vue qu'il avait eue, en 1748, de « rétablir les choses sur le même pied qu'elles estoient, il y a quatre à cinq ans $\gg .^{15}$ Il serait injuste de lui en tenir rigueur. Ni lui ni personne n'eût pu rétablir des finances de paix en temps de guerre. Car le conflit, bien qu'officiellement terminé, se poursuivait sourdement en Amérique.

En 1749, à la fois pour réduire la masse des acquits et faciliter le commerce intérieur de la colonie, la Cour autorise l'intendant à lancer une émission de 280.000 livres de monnaie de cartes, ce qui en porte le total à un million. ${ }^{16}$ Malgré cette mesure, Bigot ne peut faire mieux que de maintenir les dépenses de 1749 au niveau de celles de l'année précédente. En 1750, elles atteignent presque la somme de celles de 1747. En 1751, elles dépassent largement tout ce qu'on a vu jusqu'alors, puisqu'elles se chiffrent par trois millions et demi. Le gouvernement métropolitain exprime tous les ans sa surprise et sa colère de voir le Canada engouffrer ainsi les millions de la Marine. De son côté, le gouvernement colonial s'explique plutôt mal, souligne avec raison la persistance avec laquelle les opérations militaires s'enchaînent dans l'Est et dans l'Ouest et dénonce avec une certaine maladresse les «abus » que commettent des subalternes apparemment insaisissables. ${ }^{17}$

En réalité, il se produit des malversations, mais ce qui grève vraiment le budget des dépenses, c'est la nécessité de résister aux pressions que l'empire américain de l'Angleterre exerce sur les frontières trop vulnérables de la Nouvelle-France. Ainsi, en 1751, alors que l'état du roi fait un fonds de 130.000 livres en vue d'acheter des marchandises et des vivres dans la colonie, les besoins du service requièrent pour cet article la somme de 1.601.461 livres. Bigot justifie de la façon suivante cet énorme

15 Bigot à Maurepas, 7 novembre 1748, H. A. Innis, éd., Select Documents in Canadian Economic History (Toronto, 1929), 415 .

16 Ordonnance du roi du 18 avril 1749, Shortt, 2 : 774-782.

17 Sur les explications du gouvernement canadien et les observations du gouvernement français, voir François Bigot, administrateur français, 2: $37-50$. 
excédent de dépense: le détachement de 300 miliciens expédié à Beauséjour a entraîné de grands frais; les convois dépêchés dans les pays d'en haut et les présents qu'exigent les alliés indigènes ont «épuisé » les magasins du roi à Montréal; il a fallu, enfin, nourrir les Acadiens et les troupes de la rivière Saint-Jean. L'état du roi assigne un fonds de 20.000 livres au transport des munitions et des vivres à l'intérieur du pays et aux frontières; ce service a coûté 323.563 livres. On a dû destiner près d'un quart de million à l'établissement des postes frontaliers de l'Acadie, bien que le budget n'ait pas prévu cette dépense. Le même budget limite à 20.000 livres des débours à faire dans l'Ouest « à l'occasion des mouvements des sauvages 》; les comptes relatifs à cet article n'en montent pas moins à 135.596 livres. En 1751, les excédents de dépense se retrouvent aux mêmes postes que pendant la guerre. ${ }^{18}$ Il ne s'agit pas d'une coïncidence. L'intendant en a prévenu la Cour en 1750: «Les dépenses sont immenses et hormis d'abandonner Tout aux Anglois, Je ne prévois pas que cela puisse estre autrement. $\gg 19$

Que penser de la mauvaise humeur du gouvernement royal à propos de la masse de traites qui lui arrivent tous les ans du Canada ? En 1749, par exemple, les deux millions que la colonie coûte à la France représentent $7.9 \%$ des dépenses de la Marine, mais $23.2 \%$ de celles des colonies. ${ }^{20}$ Il est vrai que, la même année, le total global des dépenses ordinaires du royaume se chiffre par 220 millions. ${ }^{21}$ Le Canada reçoit donc un peu moins d'un pour cent des sommes que l'État métropolitain engage dans les œuvres de la nation. Vus dans cette perspective, les grands gestes réprobateurs de la Cour perdent beaucoup de leur effet. Le Canada, que l'on représente comme ruineux, ne prend qu'une parcelle des ressources du gouvernement français. Reconnaissons

\footnotetext{
18 «Bordereau général de Recette et dépense faites en Canada pendant L'année mil sept Cent Cinquante-un 》, AC, C 11A, 119: 406-429.

19 François Bigot, administrateur français, $2: 37$.

20 Voir A. Duchêne, Histoire des finances coloniales de la France, 44.

21 M. Marion, Machault d'A rnouville. Etude sur l'histoire du Contrôle général des finances de 1749 à 1754 (Paris, 1891), 17.
} 
néanmoins que cette parcelle s'est multipliée par cinq depuis 1711 , alors que la colonie n'absorbait que $0.17 \%$ des dépenses du royaume. ${ }^{22}$ En comparaison des dépenses totales de la France, celles du Canada restent modestes. Elles ne paraissent fortes que dans le cadre de celles de la Marine. C'est que le budget de la France est mal distribué. On conçoit mal qu'en 1749, un grand État comme la France, pris dans un conflit qui l'oppose à la première puissance maritime et coloniale $d u$ monde, ne destine que $11.7 \%$ de ses dépenses à la marine et que $4 \%$ aux colonies. La même année, le seul article des «menus plaisirs » du roi engouffre 2.700.000 livres, un demi-million de plus que la part du Canada. La même année encore, le voyage que Louis XV fait au Havre «pour l'important objet d'y manger du poisson » coûte un million. ${ }^{23}$ En 1754, au moment où la guerre de la Conquête est commencée en Amérique, les dépenses du gouvernement français montent à 232.701.000 livres; celles de la Marine, à 21.785.968 livres; celles des colonies, à 16.388.710 livres et celles du Canada, à 4.466.021 livres. En 1754, la Maison du Roi absorbe 6 millions; celle de la Reine, 1.800 .000 livres et celle de la Dauphine, 1.600.000 ; les «menus plaisirs » coûtent à la nation 4.600 .000 livres. ${ }^{24}$ Ce n'est pas scandaleux. C'est ridicule.

\section{CONCLUSION}

Des pages qui précèdent, il est permis de dégager quelques faits. Celui qui frappe le plus est peut-être la stabilité relative des recettes et des dépenses de l'État colonial durant la plus grande partie du demi-siècle que nous avons passé en revue: stabilité que l'établissement de moyennes décennales (tableau V) met en pleine lumière.

22 Voir G. Frégault, «La Colonisation du Canada au XVIII e siècle 》, Cahiers de l'Académie canadienne-française, 2: 66 .

23 Marion, Machault d'Arnouville, 372s.

24 Ces chiffres sont tirés de Duchêne, Histoire des finances coloniales de la France, 44 et de Marion, Machault d'Arnouville, 388s. 
TABLEAU V

Moyennes décennales des recettes et dépenses

Années

Recettes

Recettes

1711-1720

470.747 livres

extraordinaires

Dépenses

1721-1730

425.433 "

115.455 livres

$1731-1740$

466.605 “

106.776 “

483.478 livres

1743-1751

742.398 “

110.085 “

469.200 "

504.499 “

284.502 “

2.161 .470 “

Vers la fin de la période à laquelle la guerre de 1744 met un terme, les recettes du gouvernement colonial, après avoir fléchi durant dix ans, se rétablissent à peu près au niveau qu'elles avaient atteint dans les années consécutives à la guerre de la Succession d'Espagne. Pendant ce temps, les dépenses décrivent une courbe sensiblement parallèle à celle des recettes, bien que l'écart entre celles-ci et celles-là s'élargisse un peu plus entre 1721 et 1730 que durant les dix ans qui ont précédé et les dix ans qui suivront. Après 1744, il est naturel que l'état de guerre provoque une hausse des dépenses que suit, de très loin, une augmentation des recettes.

Contrairement à ce qu'on pourrait supposer, les mises de fonds de la métropole n'augmentent pas en raison du peuplement et du développement économique de la colonie. Jusqu'en 1744, elles diminuent pendant que s'accroissent la population et les productions du Canada (tableau VI).

TABLEAU VI

Moyennes décennales: finances, population, productions

Années

$1711-1720$

$1721-1730$

$1731-1740$

$1741-1750$

Dépenses
du roi (a)
368.023 livres
362.424 “
394.914 “
.876 .968 “

Fourrures (b) (valeur-argent)

957.051 livres

962.750 "

1.286.285
Blé

(minots) Population (c) $247.764 \quad 21.920$ hab. $361.425 \quad 28.555$ " $528.559 \quad 39.627$ “ c. 53.000 “

(a) Pour obtenir ces chiffres, nous avons soustrait le total des recettes extraordinaires de celui des dépenses.

(b) Nous avons pris pour base de nos calculs les chiffres donnés par Salone, 397s.

(c) Les chiffres de cette colonne, comme ceux de la quatrième, proviennent des recensements conservés dans la série $\mathrm{AC}, \mathrm{G} 1$, vol. 461 . Le dernier chiffre (53.000 hab.) est conjectural; il est basé sur le taux moyen de l'augmentation de la population au cours des périodes précédentes. 
Pour mieux saisir la valeur de ces chiffres par rapport à la population canadienne, il n'est peut-être pas mauvais de réduire ces moyennes décennales à des moyennes calculées par tête (tableau VII). Combien chaque Canadien coûte-t-il au roi ? En même temps, combien chaque Canadien produit-il de blé et vend-il de fourrures ? En vue de rendre ces chiffres plus significatifs, risquons-nous, sous toute réserve, à mettre un prix au blé; en attendant le jour où la courbe du prix de cette denrée sera établie, nous adoptons l'évaluation qu'en a faite M. Marcel Trudel, soit 4 livres le minot. ${ }^{25}$

TABLEAU VII

Moyennes par tête: dépenses, blé, fourrures

Années

1711-1720

Dépenses

Blé

Fourrures

1721-1730 17 livres $4 \mathrm{~s}$.

46 livres

1731-1740

12 " 13 “

50 “ 8 s.

33 livres $10 \mathrm{~s}$.

1741-1750

9 “ 19 “

53 “ 4 “

24 “ 6 “

c. 35

8 “

c. 24

5 “

Ces données nous enseignent que, de 1710 à 1740 , la colonie ne coûta pas aussi cher au gouvernement français que celui-ci prit l'habitude de le déplorer. Salone estime à un demi-million les dépenses annuelles que le maintien du Canada imposait à l'tat métropolitain. Le chiffre est fort: de 1710 à 1740 , le pays entraîna, année commune, un débours de 375.000 livres. C'était une bagatelle. Le souverain eût pu prendre cette somme sur le fonds de ses «menus plaisirs » et avoir encore de quoi s'amuser énormément. On comprend que Salone ait écrit: «Et quand même la Nouvelle-France continuerait, pendant une ou deux générations, à prélever sur les ressources de l'ancienne un demimillion chaque année! Pour assurer à notre race sa place dans le monde, pour lui conserver la moitié du continent le plus approprié par la richesse de son sol, par la salubrité de son climat, à l'expansion européenne, à quels sacrifices un gouvernement prévoyant, une opinion publique éclairée ne devraient-ils pas se résigner ? ${ }^{26}$ Toute la question est de savoir si, au XVIII ${ }^{e}$ siè-

25 Le Régime seigneurial (Ottawa, 1956), 14.

${ }^{26}$ La Colonisation de la Nouvelle-France, 425. 
cle, un gouvernement pouvait être assez prévoyant et une opinion, assez éclairée pour soutenir une colonie qui n'avait rien d'une île à sucre et qui, après une occupation d'un siècle et demi, n'avait encore pour elle, vue de la métropole, que des promesses d'expansion.

A comparer les dépenses des années de paix à celles des années de guerre, un autre fait apparaît. Il est curieux. En temps ordinaire, la Cour accorde au Canada des budgets insuffisants. Il en résulte des déficits qu'elle met des années à combler. Les habituels excédents de dépenses sont rejetés d'un exercice sur l'autre et donnent lieu à des émissions de monnaie fiduciaire. Dans les expériences monétaires que l'État colonial est forcé de mettre sur pied, les risques sont du côté des Canadiens: c'est ce que laisse prévoir la demi-banqueroute de 1714, c'est ce que confirmera la débâcle financière de 1759. En un mot, ce que le gouvernement royal fournit à la colonie se présente trop souvent sous forme de secours limités et tardifs. Survient une guerre. Les dépenses augmentent. La France ne veut pas perdre le Canada. Les mises de fonds qui eussent paru exorbitantes en temps de paix sont plus aisément admises en période d'hostilités. La défense du territoire et les impératifs d'une politique continentale obligent l'État colonial à mettre les postes en état, à combler de présents les indigènes, à lancer dans toutes les directions des corps expéditionnaires, à ériger des fortifications, à construire plus de bateaux, à employer et à nourrir plus de monde. La vie économique en reçoit une impulsion décisive.

Fait curieux, avons-nous dit, mais empressons-nous d'ajouter qu'il n'a rien d'exceptionnel. Dans les colonies britanniques aussi, les conflits internationaux s'accompagnent d'injections de capitaux qui stimulent fortement l'économie. A la veille de la guerre de la Conquête, on verra, dans le New-York, des négociants évoquer avec nostalgie la guerre de la Succession d'Autriche, belle époque où l'argent était abondant, les prix élevés, le crédit facile et les marchés actifs. ${ }^{27}$ Et l'on n'ignore pas que les années 1755-

27 V. Harrington, The New York Merchant on the Eve of the Revolution (New-York, 1935), 289-291. 
1760 , en raison des sommes fantastiques que l'Angleterre jette dans le conflit, auront été une période d'euphorie économique pour l'Amérique britannique. ${ }^{28} \mathrm{Du}$ reste, ne savons-nous pas d'expérience que la guerre a eu des effets analogues bien après le XVIII' siècle ?

Ces constatations nous aident à préciser un phénomène que nous avons déjà noté dans une autre étude. Entre 1720 et 1743, les témoignages ne manquent pas sur la pauvreté du Canada. Charlevoix déclare en 1720 que les habitants vont «tout nuds », en particulier ceux des agglomérations éloignées des villes, parce qu'ils ne peuvent pas vendre aux citadins «tout le surplus de leurs denrées ». L'année précédente, l'évêque de Québec avait écrit: 《Les misères de la colonie augmentent tous les jours. » En 1724, Vaudreuil s'apitoie sur les souffrances des pauvres. En 1733, Hocquart estime que la plupart des Canadiens « ont besoin de l'industrie la plus ménagée pour vivre ». En 1738, Dosquet ne parle pas autrement. En 1743, Hocquart représente la colonie comme un pays «misérable». Et ce n'est là que le dessus du panier. ${ }^{29}$

Mais voici qu'on va atteindre le milieu du siècle. Maintenant, les témoins changent de ton. Qu'il s'agisse de Kalm, de Franquet, de J. C. B., de Montcalm, ils soulignent tous l'édification de fortunes importantes, l'activité du commerce et l'aisance des habitants. Montcalm compare ceux-ci à des «petits gentilshommes de France ». Bougainville dit qu'il ne faut pas les traiter de paysans. D'autres évoquent le luxe des vêtements dont les femmes se parent. Nous avons vu Pontbriand conseiller l'imposition d'un droit d'entrée sur la soie. Franquet, comme Salone le rappelle finement, se trouve si bien reçu par un habitant de Lachenaie qu'il se prend à réfléchir, en sortant de chez son hôte, que le roi devrait bien «charger un peu » des gens qui vivent aussi largement. $^{30}$

Faut-il renvoyer dos à dos ces deux groupes d'observateurs ? Retenons, au contraire, ce qu'ils rapportent, mais sans perdre

${ }^{28}$ La Guerre de la Conquête, 283-285.

29. La Civilisation de la Nonvelle-France, $70 \mathrm{~s}$.

30 La Colonisation de la Nouvelle-France, 399-401. 
de vue le moment où ils parlent. Les premiers voient la société canadienne à l'époque où elle reçoit peu du roi. Non pas que celuici ne lui donne rien: entre 1730 et 1740 , il soutient les forges de Saint-Maurice, il assure le succès de la construction navale par ses primes et plus encore par ses commandes, enfin, il contribue à l'expansion de l'agriculture en réservant à la colonie le marché de Louisbourg. Cependant, malgré les subventions dont l'industrie bénéficie, les dépenses de l'État ne cessent de diminuer par rapport à la population, et le progrès de l'agriculture ne compense pas le recul du commerce des fourrures.

Après 1743, les témoignages des contemporains concordent tout aussi bien qu'avant cette date avec les faits qui se dégagent du présent essai. Le gouvernement français destine maintenant des sommes importantes à la défense du Canada. Proportionnellement à la population du pays, il y investit 3.5 fois plus d'argent entre 1740 et 1750 qu'il n'a fait entre 1730 et 1740 . En même temps, la traite des pelleteries se maintient. Les revenus de la terre augmentent-ils ? On n'en sait rien. Mais, même s'ils retombaient au niveau d'où ils étaient partis entre 1710 et 1720 , la perte qui en résulterait serait très largement récupérée par les dépenses de l'État. Une conclusion s'impose donc: la différence entre les sommes que la France engage dans son entreprise coloniale avant et après 1743 se traduit par une variation nettement marquée dans le bien-être du Canada.

C'est ainsi qu'apparaît au grand jour le rôle essentiel que l'État métropolitain a joué dans la formation du Canada. L'ampleur de ce rôle - dont la nécessité s'affirme aussi décisive quand le gouvernement français le joue bien que lorsqu'il le tient mal - procède de causes précises et entraîne des conséquences certaines. Analyser les unes et les autres nous entraînerait trop loin. Contentons-nous d'en indiquer les principales. Depuis Parkman, il est devenu banal de souligner le dirigisme économique qui s'est manifesté au Canada. A considérer l'action directe et indirecte de l'État dans le financement des entreprises coloniales, ne serait-il pas inconcevable qu'il en fût allé autrement? D'autre part, ce n'est pas par caprice, ce n'est pas même parce qu'il y tenait que l'État a fait sentir partout sa présence. Cette politi- 
que s'est imposée à la France comme elle s'est imposée au Canada, et la France s'y est résignée - d'assez mauvaise grâce parce qu'aucune autre politique ne s'offrait à elle: la façon dont elle en a assumé les frais suffirait à le prouver.

Coloniser, c'est essentiellement transplanter des hommes organisés en société dans un territoire susceptible d'être mis en valeur. Avec les hommes, passent les idées, les techniques, les capitaux, qu'accompagnent la mise en place d'une politique, l'aménagement d'une économie et le développement de structures sociales. Le peuplement apparaît comme le premier des facteurs qui conditionnent la croissance d'une colonie. Il peut être favorisé dans la métropole par des pressions économiques et idéologiques qui déterminent une émigration et dans le territoire à coloniser, par la richesse et la variété des ressources exploitables. En d'autres termes, les populations coloniales ne s'établissent pas et n'augmentent pas en raison de l'importance des populations métropolitaines: à ce compte, c'est la France qui eût jeté le plus de monde en Amérique; elles se fortifient en raison des besoins qui poussent des groupes d'hommes à essaimer et, en même temps, en raison de l'attraction qu'exerce un pays neuf sur ces mêmes hommes. Ces deux séries de causes peuvent jouer fortement ou faiblement. Dans le cas où elles jouent fortement, la colonisation donne l'impression de se développer en vertu de son propre dynamisme interne, sans qu'il se révèle indispensable que l'État métropolitain y tienne un rôle prépondérant. Dans le cas où elles jouent faiblement, il faut que le gouvernement de la mère-patrie s'inquiète davantage d'organiser le peuplement de la colonie, de défrayer son administration, de financer son industrie, de fournir des débouchés (intérieurs et extérieurs) à son commerce.

Dans un cas comme dans l'autre, on voit à l'œuvre le couple Etat-Société: il serait faux de dire que la Société agit seule dans le premier et l'État seul dans le second. Ce qui est vrai, c'est que, dans le premier cas, la Société verse le plus lourd de la contribution du couple (parce qu'elle y trouve son compte) et que, dans le second, la part de l'État doit être relativement plus large (parce que la Société, dont l'apport est pourtant réel, n'éprouve pas d'intérêt à faire davantage). Est-ce à dire que la colonisa- 
tion soit de meilleure qualité lorsqu'elle est surtout l'œuvre de la Société que lorsqu'elle est surtout l'œuvre de l'État? On ne voit pas pourquoi il en irait ainsi. Il ne faut pas oublier que l'État et la Société forment vraiment un couple et que, par conséquent, ce sont les mêmes hommes, les mêmes idées et les mêmes techniques que l'on voit à l'œuvre au sein de ce couple. Poser le problème de la qualité de la colonisation, c'est poser un faux problème. C'est la masse de la colonisation qui compte. Il est clair que la colonisation sera d'autant plus massive qu'elle sera plus rentable et que le rôle de l'État sera d'autant plus restreint par rapport à celui de la Société que les difficultés de la mise en œuvre seront moins grandes et plus forts les profits immédiats à réaliser. Aussi, quand l'État se voit contraint de faire beaucoup, est-ce mauvais signe: non pas parce que l'État fait mal ce qu'il fait, mais parce qu'il est naturel qu'on lui abandonne les entreprises déficitaires.

Ainsi s'expliquent l'attitude du gouvernement français à l'égard du Canada, le choix des méthodes qu'il a appliquées à l'aménagement du pays et le sort éventuel de la colonisation qu'il y a mise sur pied.

GuY FRÉGAULT

Université de Montréal 